\title{
Competent treatment of coronavirus
}

\section{(Analysis of literature sources and own research - level of evidence-4)}

Dmitrieva Elena Germanovna

Charity Pharmaceutical Clinic, Clinical Pharmacist, candidate of biological Sciences, specialty-Clinical Pharmacology, Russia.

Corresponding Author: Dmitrieva Elena Germanovna, Charity Pharmaceutical Clinic, Clinical Pharmacist, candidate of biological Sciences, specialty-Clinical Pharmacology, Russia Email: cyrix2003@mail.ru

Received date: September 29, 2020; Accepted date: October 05, 2020; Published date: November 07, 2020

Citation: Dmitrieva E. Germanovna (2020) Competent treatment of coronavirus J, Pharmaceutics and Pharmacology Research 3(2); DOI: 10.31579/ 2693-7247/017

Copyright: (C) 2020, Dmitrieva Elena Germanovna, This is an open access article distributed under the Creative Commons Attribution License, which permits unrestricted use, distribution, and reproduction in any medium, provided the original work is properly cited.

\section{Introduction}

The main biological feature of the virus is its high contagiousness, which is many times higher than that of viruses that cause various acute respiratory infections, including influenza. The root cause is reduced immunity as a result of long-term illiterate vaccination of mankind by generations (author's note). The coronavirus is transmitted from person to person and from animal.

\section{Types of coronaviruses}

\section{There are four types of seasonal coronaviruses that cause} respiratory infections:

HCoV-NL63, HCoV-229E, HCoV-OC43, and HCoV-HKU1. Most often, these infections are asymptomatic. However, reinfections were most often observed 12 months after initial infection, and in some cases six to nine months later. This applies to all four viruses studied. Hence, the authors conclude that immunity against the new SARS-CoV-2 coronavirus is likely to be short-no more than a year. In winter, people in temperate countries are more likely to become infected with this family of viruses. According to the authors, the same picture should be expected for SARS-CoV-2, when it becomes seasonal after the pandemic. A longterm study of seasonal human coronaviruses has shown that the immunity of those who have had an infection, as a rule, does not last long - from six months to a year. The four viruses studied belong to different groups: HCoV-NL63 and HCoV-229E are alphacoronaviruses, while HCoVOC43 and HCoV-HKU1 are betacoronaviruses. They use different receptors to enter cells. HCoV-NL63 enters the cell through the ACE2 receptor, as does SARS-CoV-2, the rest through other receptors. But the nature of the immune response is very similar for everyone. You should rely on natural immunity, and not engage in vaccination (author's note).

A key factor for the transmission of SARS-CoV-2 coronavirus is the rate of evaporation of moisture in the air.

Using theoretical modeling and hydrodynamic experiments, experts studied the effect of humidity, ambient temperature, and wind speed on the spread of tiny drops of saliva containing virus particles. It was found that the viability of the virus is significantly reduced at high temperatures and low relative humidity due to the high rate of evaporation. In hot weather with high humidity, the probability of transmission of coronavirus remains high. The findings explain why the pandemic intensified during July in various densely populated cities around the world with hot and humid climates.

\section{Methods diagnostic}

Laboratory diagnostics of coronavirus infections, including SARS, is performed by:

1. By isolating virus cultures and identifying them.

2. Either by determining virus-specific antibodies and increasing their titer in paired serums using various serological reactions or using DNA and RNA probes, PCR. However, all the proposed test systems for SARS diagnostics require additional study of their specificity. Their reliability is up to $70 \%$.

\section{The author proposes to look at the dynamics:}

1. Biochemical analysis - CRP, rheumatoid factor, ferritin, serum iron, zinc, and thymol test, AST, ALT, creatinine phosphokinase, d-dimer in venous blood-increased ferritin, decreased zinc, increased inflammation.

2. Immunograms of 2 levels of complexity and their analysis (reduction of $\operatorname{IgA}$ and secretory $\operatorname{IgA}$ )

3. Protein fractions (decrease in total protein, hypoalbunemia, and rarely-hypoglobulinemia)

4. Clinical analysis (blood from the finger) - leukopenia, lymphocytopenia, thrombocytopenia, acceleration of ESR.

Signs of coronavirus infection in the biochemical blood analysis:

1. Increased levels of liver enzymes ALT, AST or ALAT, ASAT (full name alanine-aminotransferase and aspartate-aminotransferase or transaminase);

2. Increased C-reactive protein (abbreviated CRP) - increases with any inflammatory processes in the body;

3. An increase in the level of ferritin in the blood (hyperferritinemia) indicates a severe course in Covid-19. This is a marker for viruses and bacteria entering the body. Also, the level of ferritin is increased in hemochromatosis and excessive iron accumulation syndrome, sepsis, antiphospholipid syndrome (abbreviated AFS);

4. An increase in the level of troponin in the blood occurs in patients with ARI (acute respiratory diseases). Troponin indicates damage to the myocardium (heart muscle) and in coronavirus infection, if there is no suspicion of damage to the myocardium, the level of troponins is not determined; 
5. The level of D-dimers (fibrin breakdown products) is increased. Ddimers in high concentrations appear in the blood after the destruction of a blood clot and a high level of D-dimers indicates the presence of blood clots. In patients with severe Covid-19 (severe pneumonia, acute respiratory distress syndrome (ARDS) and other complications of CVI), the level of D-dimers is abnormally elevated. Attention! In any case, PCR smears from the anus in the case of children may be more informative for finding infection than PCR flushes from the nasopharynx (author's note).

6. In dynamics (at the initial treatment of the patient and after 2 weeks), a blood ELISA is performed for the presence of antibodies to the coronavirus. The minimum increase in the antibody titer to confirm the diagnosis is twofold. The most informative ELISA becomes 10 or more days after the first clinical manifestations.

7. For the purpose of differential diagnostics, a bacteriological examination of feces and a coprogram are used.

8. Roentgenography of organs of a thorax in 2 projections

9. Multispiral computed tomography of the lungs is used less frequently (to exclude similar pathologies).

10. Markers-interleukin-6 (Christoph Messner, 2020).

11. According to the General and biochemical blood analysis $+\mathrm{d}$-dimer level, the diagnosis of Covid-19 is not made.

It is recommended to look at the soluble complex of fibrin monomers (RFMC) - this is the most sensitive test. This is what is included in the concept of coagulogram. But, unfortunately, this test was not used everywhere during the epidemic. Everyone has looked at the D-dimer, but it works in very severe conditions, and the rfmc is much more sensitive than the D-dimer. The D-dimer may be normal, and the rfmc may be elevated.

The diagnosis is made by a PCR smear from the nose (if the coronavirus RNA is found in the material) + Covid-19 antibody test. Sometimes it is based on a typical picture of viral pneumonia in a CT scan of the chest. Changes in the lungs in viral pneumonia are poorly visible on a normal $\mathrm{x}$ ray. The $\mathrm{x}$-ray picture is usually characterized by unilateral interstitial lesion, or bilateral focal drain pneumonia.

UAC and BAC are included in the mandatory list of examinations for suspected coronavirus infection and confirmed diagnosis of Covid-19. The General blood test for viral and bacterial infections looks different. What in everyday life is called viral blood or viral analysis looks exactly as described above.

Differential diagnosis is performed with other acute respiratory infections, influenza, $\mathrm{Ku}$ fever, pneumocystosis, tuberculosis, legionellosis, ornithosis, mycoplasmosis, bacterial rhinopharyngitis, bronchitis, pneumonitis and pneumonia.

It is necessary to differentiate this pathology with viral diarrhea, salmonellosis, food toxicoinfections, dysentery, enterovirus infection.

12. Pulse oximetry-oxygen saturation-low saturation-gives shortness of breath.

\section{Discussion}

\section{My opinion of an Expert Clinical Pharmacist is that it is pneumonitis}

Pneumonitis is an inflammation of the alveolar walls and interstitial lung tissue of mainly non-infectious origin, which leads to scarring of the alveoli and fibrous changes in the supporting structures of the lungs. The difference between pneumonitis and pneumonia is clearly visible on $\mathrm{x}$ rays. In pneumonitis, the pulmonary pattern is enhanced, there are darkening in the lower part of the lungs, and in pneumonia, the darkening foci have uneven contours in various parts of the lungs. Features in children-complications of pneumonia and pneumonitis in young patients appear immediately after the disease. A child who is considered to have recovered may feel ill.

\section{The mechanism of development of pneumonitis is twofold.}

First, red blood cells settle, causing fibrin to clot and fall out, and intracellular penetration of the coronavirus joins, then a cytokine storm develops, when all the active substances that destroy the cells affected by the virus gather in the inflammation zone. Plus pulmonary edema caused by complications from the heart and blood vessels (author's note). Edema of the lungs is removed so-the needle is inserted into a vein and the blood goes down into the basin. This is the fastest way to relieve pulmonary edema (author's note). If you enter a diuretic-Furosemide (Lasix) into a vein, you may not have time for a latalny outcome!!!

Smoking is a serious risk factor for coronavirus, because when Smoking, hemoglobin is increased and iron in the body is a compensatory reaction to hypoxia (author's note).

Invagination angiogenesis (IA) is the way the body compensates for thrombosis and damage to blood vessels.

Damaged blood vessels may also be at the root of other problems observed, such as leg COVID, children with Kawasaki, stroke, and other seemingly unrelated problems observed with COVID-19.

This study shows the need for additional studies of angiogenesis and vascular effects of COVID-19.

Examples of writing diagnoses in medical records and medical records (author, 2020):

1.SARS is always a severe course ( $L$-form) with or without transient hemochromatosis, eosinophilia or without it

\subsection{9-nCoV-S-form}

3.Intestinal coronavirus, gastroenteritis

4.SARS complicated by Kawasaki disease (typical for children 1-2 years old)

\section{MERS}

\section{Pathological anatomy-named leading to death in coronavirus-abnormal pathology}

Hundreds of blood clots are found in the lungs of patients who have died from coronavirus. Abnormal blood clotting leads to a fatal outcome. It was patients with this syndrome who were more likely to suffer from coronavirus and end up in intensive care.

In addition to the affected coronavirus pneumonia pulmonary alveoli, in the lungs there were hundreds of microthrombi. This deviation is not observed in other types of lung infections. The clotting disorder partly explains the occurrence of hypoxia (a drop in blood oxygen levels) in patients with COVID-19.

Abnormal blood clotting in severe coronavirus significantly increases the risk of stroke or heart attack (James O'donnell, 2020). Abnormal blood clotting in severe coronavirus significantly increases the risk of stroke and heart attack, so you need to include Thrombolytics in the treatment standards when necessary (author's note).

\section{Differences in the course of coronavirus in women and men.}

Women carry this infection more easily.

Coronavirus causes pulmonary hypoxia (a drop in blood oxygen levels). All internal organs suffer greatly from this.

\section{Women can tolerate hypoxia more easily than men.}

Science knows this, and in any diseases that are associated with acute hypoxia and serious manifestations, women survive better than men (A. Minurova, 2020). This is the envy of physiology-male and female. In addition to physiology, another factor is the lifestyle of men.

The male half of humanity is still less health-conscious and less likely to take care of chronic diseases that worsen the course of the coronavirus.

There is an accumulation of fluid inside the alveoli of the lungs, but also many other processes. 
In the past three weeks, there has been a clear increase in the number of children of all ages suffering from a systemic inflammatory disease requiring intensive therapy - Kawasaki syndrome (children 1-2-5 years old).

Caused by certain vaccine infections that have gained strength against the background of coronavirus (increased infection syndrome).

It is necessary to develop a treatment for Kawasaki disease (author's note).

\section{Immunology of coronavirus}

Generally, children have low levels of IgA and secretory IgA. Probiotics help, for example, Hilak-Forte drops and others.

These properties have viruses and, oddly enough, - anti-Retroviral prerarates (author's note), so I do not recommend using children under 1 year of life HIV-infected from HIV-infected mothers (author's note). If you do not use vaccines, and HIV-infected vaccines are not shown, then the body of children can heal itself (author's note).

Attention! I do not recommend taking anti-Retroviral drugs for the treatment of coronavirus (author's note).

\section{Opinion of the author-Expert-Clinical Pharmacist.}

Official medicine is mistaken that the pneumococcal vaccine can save from bacterial complications of the coronavirus. There is an increase in infection (increased infection syndrome) when mixed infections develop. When vaccination occurs, there are 3 possible outcomes: a surge of immunity, fighting infection, when there is a meeting of a natural virus, the immune response is sluggish, and the body often gets sick again from vaccines, the second way is complications due to the lack of antibodies, which are not formed in $20-30 \%$ of children and adults. 3 path-death. This was recognized by the Ministry of health of Russia, while blaming the responsibility of vaccination on the parents of children.

The virus weakens by infecting a person and jumping to another person. The virus mutates. But it doesn't make exact copies, but different ones to mutate. This is important to him for infection.

\section{The COVID-19 alcoholic beverage has been named to ease the flow}

The wine contains the antioxidant resveratrol, which can facilitate the flow of COVID-19.

It is noted that the coronavirus also disrupts the work of the ACE2 protein, which is present in the tissues of the lungs, heart, kidneys, and brain and is necessary for life. Resveratrol increases the concentration of ACE2 and thus reduces the severity of acute respiratory distress syndrome (ARDS) that accompanies COVID-19.

In addition, the antioxidant can suppress immune system cells by reducing the amount of cytokines. Since ARDS is caused, among other things, by an excessive immune response, resveratrol may have an additional positive effect on this complication.

Resveratrol is found not only in wine, but also in grapes, cranberries, and peanuts.

\section{The body needs healthy mucous membranes}

In this case, the person most likely will not get infected, even if there is a carrier of the infection in the same room with him.

To the mucous membranes because this is the main defense mechanism against dangerous bacteria and viruses. In fact, our local immune defenses. Now in the period of the COVID-19 epidemic, doctors advise strengthening local immunity through the restoration of the mucous respiratory tract and gastrointestinal tract. This protection is-the lymphoid tissue of the nasal mucosa and tonsils in the pharynx (there are several in the nasopharynx and they can not be removed) and the intestinal lymphoid tissue (author's note).

All mucous membranes protect a person from pathogenic bacteria, toxins and viruses. With the help of mucus. Mucus (mucin) - is needed to mechanically protect cells from «interventionists», wash them off and remove them from the body.

In addition, the mucus contains special substances-Secretory Immunoglobulins, which are able to neutralize viruses directly where they try to enter the body. This is called local immunity.

\section{Antivirus antibiotic of animal origin - Ecmolin.}

Ecmoline (Ecmolinum) - $0.5 \%$ aqueous solution of triprotamine sulfate. A solution of triprotamine sulfate is produced, in $10 \mathrm{ml}$ vials.

It is used for the prevention and treatment of catarrhal phenomena in the upper respiratory tract with flu and others

Ecmoline is administered 4-10 drops in each nostril 3 times a day or used for inhalation in the form of an aerosol- $0.5 \mathrm{ml}$ of Ecmoline in $5 \mathrm{ml}$ of isotonic sodium chloride solution.

You can add 10,000 UNITS of crystalline Penicillin to $1 \mathrm{ml}$ of the solution.

The opinion of an Expert-a Clinical Pharmacist - my rich clinical experience shows that it is necessary to introduce etiotropic drugs for the treatment of infections.

Of the most effective drugs in this group with the greatest clinical effect, but not with COVID-19 - applicable - derivatives of excicamic acid (Piroxicam, Tenoxicam and others). Only this group does not cause reverse inflammation (markers of inflammation-you need to look at the dynamics) and can be used 1 time a day at night. Then the clinical antiinflammatory effect is more pronounced at therapeutic doses or even lower than therapeutic doses.

As for Paracetamol, I do not recommend prescribing it to children. Adults on indications, and COVID-19, I consider that such indication is not. If you have a «wet cough» for prevention of atropatia from the Paracetamol (Acetaminophen) is assigned to ACC (Acetylcysteine). If the temperature is high, then Nurofen is better (author's note).

In the UK, when treating cancers, thrombolytics are often used, since the blood of such patients is condensed. I observed clinical cases in hospitals during my studies (author's note).

Combined treatment $($ pharmacotherapy + phytotherapy $)=$ rational grain for the joint use of two types of therapy (author's note).

The author developed a bloodless differential diagnosis of

hypovitaminosis (author's note).

The wave-like flow of the coronavirus-the cough disappears, then returns, as does the temperature.

When the temperature goes down-Immunomodulator-Imunofan to reduce intoxication.

\section{Up temperature-antiviral (Ribavirin).}

Schemes of Immunomodulators instead of-candles, intra/v instead of droppers. Candles from 2 years, from 1 year candles can be put in per rectum 1 time a day-the course in 3 days.

\section{Drugs that help patients-translation Inhibitors (author, 2020)}

A coronavirus infection changes human cells. To do this, scientists used a special type of mass spectrometry method mePROD to determine the rate of protein synthesis in the culture of colon cells and their number. It turned out that many viruses stop the production of host proteins in favor of viral proteins, SARS-CoV-2 only slightly affects the production of protein, but it accelerates its synthesis.

To reduce the rate of virus reproduction, scientists used translation Inhibitors that suppress the process of protein synthesis, where ribosomes, matrix RNA and transport RNA are involved. Among the substances that stop the virus from multiplying in cell culture are 2-Deoxy-D-glucose (2DG), as well as Ribavirin, an antiviral drug used against hepatitis and other infections.

There is an antiviral drug-Ribavirin. 
I used it in the early stages of the disease and got good results. When there is shortness of breath, Ribavirin helps in combination with Prednisone (5 $\mathrm{mg}$ ) or Dexamethasone $500 \mathrm{mcg}$ (the course of treatment is short - it is better to prescribe GCS in the morning in small doses). At the same time, the effect of GCS is maximum. It is not necessary to prescribe antibiotics, sulfa drugs from them in this case would not be good.

In women, during the treatment of OCD - with the simultaneous use of hormonal contraceptives - there is an increase in the action of Prednisone. Ribavirin $200 \mathrm{mg}$ (capsules) helps with bacterial infections, there are cases of helping patients (author's note).

My combination-Ribavirin + Imunofan = rational combination - only for adults and children from 2 years of age (author's note).

This combination cures. Ribavirin is not used for HIV infection!!!

Combination of Ribavirin + Imunofan = the best!!!

Short treatment regimen for coronavirus (author, 2020).

\section{For the Elderly}

1. Mexidol (Mexiprim)-5 ml intravenously-solvent-isotonic solution of $0.9 \%$ sodium chloride.

Doses are selected individually. The drug gently lowers blood pressure and destroys the coronavirus, as the virus removes oxygen.

2. For Children-candles in the rectum-Imunofan-immunomodulatorprescribed from 2 years. There are exceptions-reception from 1 year - 1 candle 1 time in 3 days. Doses are selected individually.

3. For detoxification in Adults-Imunofan - $1 \mathrm{ml}$ intramuscularly. After 3-4 days of treatment, the drug acts as a detoxifier. You can do without artificial ventilation (ventilator).

4. In severe cases, during the intoxication phase, you can use candles, intramuscular injections-the drug-Imunofan. Doses are selected individually. The course of treatment is up to 20 injections.

5. In the phase of fever - you can prescribe antiviral drugs or a combination of them (more in my book-requires publication). Doses are selected individually. During the temperature rise phase, it is better not to use Immunomodulators, since Immunomodulators can increase body temperature.

6. Analgesics-it is better not to prescribe, except Nurofen.

7. The Author has found several chemical formulas for creating a drug with a new etiotropic effect on coronavirus.

8. With an increase in the content of iron in the blood serum and ferritin, and the coronavirus has this property, since it removes oxygen from the blood and tissues, there are transient phenomena of hemochromatosis (concentration of iron in the blood). We Need Antioxidants. You can avoid heavy drugs - iron-removing, and also use plants-iron-removing (iron-removing) plants-traditional phytotherapy (sparing treatment for patients with coronavirus). These methods are up to $98 \%$ effective.

You don't need vaccination, tracheal intubation, or a medically induced coma.

\section{The study of protein L-FABP}

The study involved 41 people. In the urine of 13 patients, an increased content of L-FABP was recorded, while eight of them had a very bad condition during the week, and two needed to be connected to artificial lung ventilation.

The link between the L-FABP protein and the symptoms of people who have contracted COVID-19. This protein occurs in human urine when the body's oxygen level decreases. This means that you need Mexidol (Mexiprim). This is better than a ventilator (author's note).

\section{Conclusion}

Now Rospotrebnadzor has temporarily suspended vaccination, except for BCG in children. I believe that any vaccination should be stopped (author's note) or a medically induced coma. It was found that the coronavirus has a $3 \mathrm{C}$-like protease, which is the main enzyme in the infection. COVID-19 has a weak point and can be used to stop virus replication. A study of the disease found a $3 \mathrm{C}$-like protease, which is also known as 3CLpro. It is the main enzyme in the development of SARSCoV-2, so if you disrupt its work, the activity of the virus can be stopped. Need protease inhibitors, which can successfully stop the replication of infection. Moreover, a set of medications can stop not only the coronavirus, but also MERS-CoV.

\section{Scientists have found the smallest protein compound that neutralizes the SARS-CoV-2 virus.}

Experts in the United States conducted a rapid analysis of one hundred billion potential molecules that can bind to the coronavirus S-protein involved in infection with the pathogen. As a result, the scientists found a protein compound ten times smaller than a full-size antibody, which, merging with part of the immunoglobulin, forms the ab8 drug and acquires the functions of a full-size antibody.

Further experiments showed that even the lowest doses of Ab8 significantly reduced the number of viral particles in laboratory animals infected with coronavirus. Scientists note that the small size of the molecule increases its ability to diffuse in tissues, helps to better neutralize the virus, and also allows the drug to be administered by inhalation.

\section{List of main errors in therapy for patients with severe forms of coronavirus infection.}

Among them: excessive use of antibiotics, which increases the risk of developing superinfections, unnoticed dynamic overexposure of the lungs, not prescribed timely anti-cytokine therapy, lack of rates of Clinical Pharmacists (author's note).

In the Ivanovo region, one of the pregnant women infected with coronavirus infection died. She was 33 years old. The patient's condition deteriorated sharply, and she had to resort to emergency delivery at 32 weeks. The child is now in a clinic in Ivanovo, doctors are fighting for his life.

The woman was hospitalized in a timely manner, and also sought advice from Federal centers. Despite timely medical care, she died. According to the results of the autopsy, almost $90 \%$ of the lungs are affected, total defeat, and signs of thromboembolism. The number of pregnant women infected with COVID-19 in the region is growing.

You can use Perftoran in the intensive care unit, 2 infusions are enough to return a person from the intensive care unit to the ward (he draws oxygen on himself). But when testing a new treatment in my region, this drug was not found (author's note).

The largest proportion of people from Russia with antibodies to coronavirus was recorded in Tatarstan, Saint Petersburg, and the Tula region.

One of the Syktyvkar women has been in the Komi Republican clinical hospital for almost two weeks with suspected coronavirus.

The girl has not been home since August 7.

She told about her treatment.

Syktyvkar's Irina Yevgenieva already knew that she had been in contact with a person who had fallen ill with COVID-19 when she felt ill.

My sense of smell and taste disappeared, a slight temperature rose, and I started coughing. She notified her doctor on July 28 . The smear was taken only a week later. It turned out to be negative. Then for the first time I thought that I had passed. But the attending doctor noticed when listening to the lungs that breathing is difficult, so she sent me for a CT scan, while saying that everything is fine, that all this is done for prevention. 
However, Irina was told that a CT scan revealed that she had left-sided viral pneumonia and the girl was forced to go to the hospital.

The girl was forcibly admitted to a hospital full of patients with a coronavirus infection. Today, people are not legally savvy at all. Obediently, changing into the issued dressing gown, she followed the hospital worker. Threats of responsibility always act on law-abiding citizens, so since August 7, the girl was in the KRB.

Irina was placed in a ward with patients who already have a confirmed coronavirus infection. The girl was admitted to the hospital on Friday, so there was allegedly no medication. The nurse explained that all the medicines had run out, and since the pharmacies were closed on Saturday, there would be no treatment until Monday. Imagine, pharmacies are closed on Saturday. That's how she was left locked up without any treatment for the weekend in a unit with people whose diagnosis had already been confirmed.

The girl said that, starting from Monday, she began to be given Ingavirin, to do UFOs of the pharynx and oral cavity and inhalation. According to the patient, the procedure took at most 15 minutes all together and took place once a day.

They also gave a solution of Chlorhexidine marked gargle up to six times a day. From the very beginning, I did not understand why I was in this institution, because my smear was negative, and I did not receive any treatment. I'm just lying in the hospital, sleeping, eating, and breathing the same air as people with coronavirus. To my questions, my doctor replied that since the smear is negative, he does not want to prescribe strong therapy, so he only treats pneumonia. Let me remind you, the treatment consisted of antiviral, UFO, gargle and inhalation.

A little later, the girl discovered that the patients are in the Department of neurosurgery and their treatment is not carried out by infectious diseases, but by neurosurgeons.

Irina's second smear on the coronavirus, which was done at the hospital, came back negative. The girl breathed a sigh of relief, but, as Irina told, her doctor explained that for the discharge of such smears, two are necessary and added a drop of Grippferon to the treatment. And after another ten days in the hospital, on August 19, Irina tested positive for coronavirus.

In addition to the ward, there are 15 other wards on the floor with a capacity of three people. Toilet and shower for the entire Department, which are washed with care and do not ventilate. There are no masks, they give one mask for the entire stay in the hospital. The clinical picture is in all colors, and what do you think, what was the smear after 10 days of stay in a Department full of people who had positive smears? That's right. It came back positive. In response to my requests to explain how this could have happened, the doctor said that it was unlikely that she could have been infected in the hospital, that the virus could simply have been located just below the place where the swab was taken.

Almost two weeks Irina is in the hospital and only one day of them she is sick with coronavirus. The girl said that her «treatment» did not change even after that.

Now, sitting on the bed, on the holey bed linen, in cold chamber, one robe, which is issued at receipt, I realized what mistake I had made when obediently got dressed, and followed by the messenger of hell, which led me to a place where you can't get out. I still do not understand why the doctors are so indifferent, do not have a sense of tact, telling you that you will have to stay here in a good way for another two weeks, and, most likely, that you are now in this place because of the fault of the doctors, because of their negligence and connivance. There is an administrative responsibility for walking around and infecting others with the «crown». And is there a responsibility to understand the whole situation and quietly watch from the outside as a healthy person turns into a sick person? The girl hopes that her story will help people understand how important it is to prevent infection, how important it is to know their rights.

I really want to avoid making mistakes and avoid infection at all. I ask you to take all necessary measures for this, because if you get sick, you are more likely to face the same thing that Irina had to face. It is time to recognize the fact that our hospitals do not treat, but cripple. We are all told that Russia, or rather, in this case, the Komi Republic, is fighting the coronavirus, and that doctors use the most powerful and reliable drugs to cure patients. I had a "happy" chance to test the entire system on myself, and therefore I think it is necessary to convey this to people.

\section{Named products that help avoid the second wave of the virus}

The second wave of the virus will help to avoid vitamin D. This means that the diet should include products containing this vitamin. Scientists conducted a study involving 500 Americans and found that people with vitamin $\mathrm{D}$ deficiency were twice as likely to get coronavirus.

The daily value of vitamin D is from 2.5 to 5 micrograms. Vitamin D synthesis occurs only with sufficient ultraviolet radiation. This vitamin is obtained from food. We are talking about products such as mushrooms, sea fish, seafood, egg yolks, cereals, cheese and fish oil.

Vitamin D plays a key role in the human immune system. Vitamin D regulates mineral metabolism and improves muscle tone. It is important for teeth and bones, as well as for the healthy functioning of the thyroid gland. In addition, vitamin D provides blood clotting. This substance stabilizes blood pressure and normalizes the heart rate, as well as strengthens the nervous system and prevents the development of cancer cells. The need for vitamin $\mathrm{D}$ is compensated by vitamin D3 (cholecalciferol), which is formed in the skin from provitamins under the influence of ultraviolet rays of sunlight.

You should also pay attention to vitamins a, B, and C. The expert recommends adding to the diet foods containing zinc, which is responsible for metabolism and immune responses (there is a mention in the text). Zinc is found in chicken meat, low-fat cheese, almonds, green peas, and pumpkin seeds. Vitamin D deficiency occurs when there is a lack of sunlight. The amount of vitamin synthesized under the influence of ultraviolet rays is also affected by the length of the light wave. The most useful is the average wave spectrum, which occurs at dawn (8-10 am) and at sunset.

Low vitamin levels are also caused by high air pollution (city dust, industrial emissions, and other air pollution factors block the access of ultraviolet rays. This is why rickets is so common in children in industrial cities in Asia and Africa. Also, the cause of vitamin deficiency may be naturally dark skin (the stronger the pigmentation, the less vitamin $\mathrm{D}$ is produced under the influence of sunlight) and old age (over the years, the skin is less able to synthesize vitamin D).

The author has developed a differential diagnosis of hypovitaminosis and microementosis, but has not yet been published (author's note).

\section{Post COVID-19 syndrome}

Not everyone is affected by this syndrome, but about $10-12 \%$ of those who are ill. What is it? My opinion is as follows. Covid affects the Central nervous system, peripheral nervous system, and autonomic nervous system. We have, as you know, three nervous systems. With COVID-19, there is thrombovasculitis. And this thrombovasculitis, we call it encephalitis, because it is an inflammation of the brain tissue, it does not go away immediately after the disease.

Initially, everyone attacked the lungs, because the lungs are what was seen by shortness of breath and other symptoms. But among all patients with covid, severe lung damage is only a few percent. The main mass was ill without such lung damage. Unfortunately, many of them had brain damage. This is shown in the following way. For example, a temperature violation. Not only increase, but also decrease: 35.5 or 36.0. And this can persist for a long time. This temperature rostkoviana syndrome does not adjust.

Then - whensyndrome marked severe headaches. Weakness - and many describe such weakness that they literally can't get out of bed. People with an active lifestyle, running several kilometers a day, they can not leave 
the house. Also, when syndrome may be loss of smell. Loss of sense of smell is not as common as weakness.

Respiratory disorders, a feeling of lack of air, spasms in the chest without pronounced lung damage. Some patients have skin lesions, various rashes, and some hair begins to fall out.

There are also mental disorders: tearfulness, bad mood, depressive mood, suicidal thoughts. But psychiatric problems can be exacerbated by infection. And if this is an isolated problem from all the other symptoms, then this is a mental problem. And if combined with other symptoms, it is a brain lesion.

Understanding which people are more prone to postavenia syndrome, not now. People who lead an active lifestyle notice this better than those who lead a passive lifestyle.

\section{Vaccination in Russia}

Approximately one in seven volunteers vaccinated against coronavirus complained of unpleasant sensations - muscle pain, weakness, fever.

Vaccinations against SARS-CoV-2 were made during post-registration tests of the Sputnik V vaccine, in which, according to the plans of the authorities, several tens of thousands of volunteers should participate. Some of them promised to inject a placebo so that the tests would meet accepted international standards.

More than 300 people were vaccinated. $14 \%$ have small complaints of weakness, muscle pain is small during the day, and occasionally there is a temperature.

In Russia, there are observations, and there are such observations abroad, that a person who has been ill releases the virus for up to 90 days. Our observations today - up to 48 days in Russia, and abroad there are up to 90 days.

In Russia, the coronavirus has infected 1073 849, recovered 884 305, died 18785 .

Vaccine developers will not vaccinate children against COVID-19 in 2020.
Patients should not be vaccinated against flu and chickenpox, which is happening now. Otherwise, there is a syndrome of increased infection when they overlap. As well as imprinting syndrome (the interaction of vaccinations with each other with the issuance of toxic phenomena). And against the background of existing treatment of Kawasaki syndrome in children, there is also the occurrence of Rey's syndrome, which is lifethreatening for children. The vaccinations themselves cause many autoimmune reactions, which is now reported in contraindications of the coronavirus vaccine. In fact, you can't do it!!! Especially for those who have already been ill.

I want to pay attention to the health of children, Russian citizens, who do not have antibodies at all for any vaccination, but only give complications and death. We need a mandatory alternative for these children and adults. Unfortunately, clinical pharmacy is still not being restored in Russia, but only this industry can prevent the interaction of drugs and vaccines. Differential diagnosis of iatropathies is necessary, when it is necessary to be able to recognize the true primary disease from iatropathies. This is very important. And this can only be done by Clinical Pharmacists. In addition, Pharmacists are now also in a coronavirus pandemic, so they also need additional payments for working in a coronavirus pandemic.

\section{Literature used}

1. Human Coronaviruses (Nidovirales, Coronaviridae): the highest level of epidemic danger /Shchelkanov M. Yu, Kolobukhina L. V., Lviv D. K. //Treating doctor-2013.

2. The Role of coronavirus infection in acute respiratory tract pathology: abstract/Muradyan A. Ya. - 2005.

3. Acute respiratory viral infections: etiology, diagnosis, modern view of treatment/Denisova A. R., Maximov M. L. //Russian medical journal-2018-N1 (2).

4. Acute respiratory infections in children: Textbook/Reshetnik L. A., Spasova Yu. S. - 2017. 